\title{
Phase Contrast HRTEM and DF-STEM Electron Tomography Reconstructions of Polymer/Layered Silicate Nanocomposites
}

\author{
Lawrence F. Drummy ${ }^{1}$, Hilmar Koerner ${ }^{2}$, Y. C. Wang ${ }^{3}$, Kelly L. Anderson ${ }^{1}$, B. L. \\ Farmer $^{1}$ and Richard A. Vaia ${ }^{1}$ \\ 1. Materials and Manufacturing Directorate, Air Force Research Laboratories \\ 2. University of Dayton Research Institute \\ 3. FEI Company
}

Montmorillonite (MMT) has shown promise as an inorganic filler material for polymer nanocomposites [1]. When properly processed into a polymer matrix, excellent dispersion (exfoliation) at the nanometer length scale can be obtained. Montmorillonite consists of plate-like aluminosilicate sheets, $1 \mathrm{~nm}$ in thickness and 100-200 nm in diameter. We have used commercially available organically modified MMT (Southern Clay, Nanocor), and processed this material into an epoxy matrix using various methods [2]. The resulting microstructures were characterized using two different high-resolution electron microscopy techniques.

Phase contrast high-resolution transmission electron microscopy (HRTEM) was used to directly image the intra-sheet lattice structure of the MMT layers. This allowed MMT to be imaged in several orientations inside an epoxy matrix (not just "edge on"). The experimental procedures to obtain high resolution lattice images from MMT sheets were defined. This included the description of defocus conditions necessary to simultaneously image the inter- and intra-sheet MMT spacings, as well as determination of the electron beam stability of MMT. Figure 1 shows a HRTEM image of a MMT sheet embedded in an epoxy matrix viewed edge on compared with a MMT molecular model. Figure 2 shows multislice image contrast simulations from polymer/MMT molecular models at different values of defocus.

Dark-field scanning transmission electron microscopy (DF-STEM) was also used to generate high contrast images of the MMT sheets at reasonably high resolution $(<1 \mathrm{~nm})$. These images are free from any defocus artifacts that can arise from phase contrast imaging. Using a high-tilt stage, data sufficient for electron tomography reconstruction was obtained. Using this technique the three-dimensional structure of the sheets can be visualized. Figure 3 shows a tilt series of an aggregation of MMT sheets in an epoxy matrix.

These microscopy methods allow for visualization of the local three dimensional structure of the MMT sheets in nanocomposites at high resolution. This data may give insight into the processes of intercalation, swelling and exfoliation of the MMT sheets during curing of the epoxy matrix.

[1] S. J. Ahmadi, Y. D. Huang, W. Li, J. Mat. Sci. 39 (2004) 1919.

[2] H. Koerner, D. Jacobs, D. W. Tomlin, J. D. Busbee and R. A. Vaia, Adv. Mat. 16 (2004) 297. 
HREM image

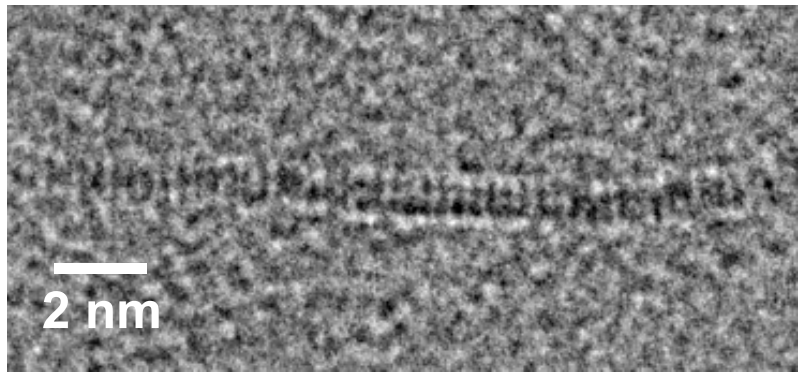

MMT model

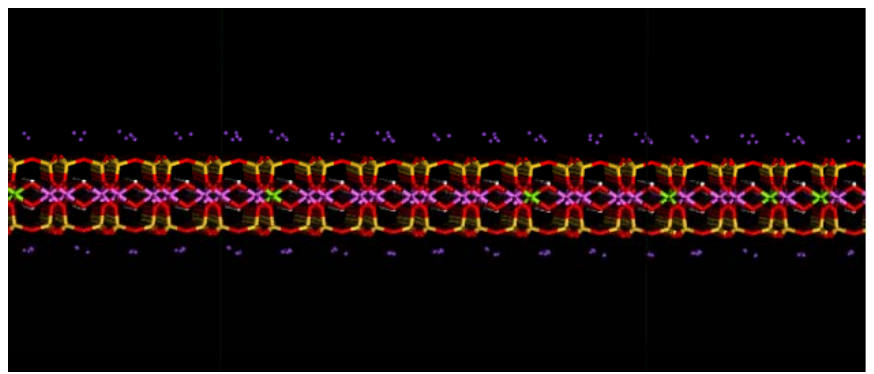

Figure 1. HREM image of a single MMT sheet in an epoxy matrix compared with a molecular model.

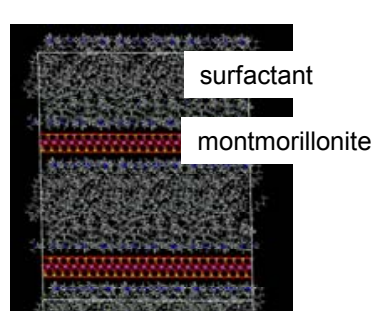

[100] orientation (molecular model)
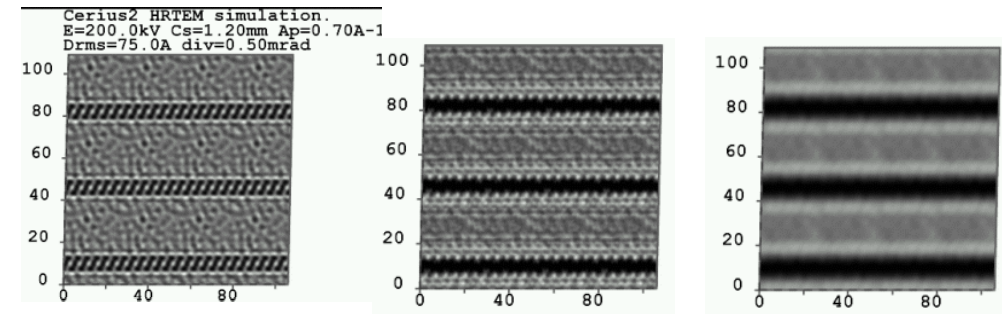

$\Delta \mathrm{f}=-55 \mathrm{~nm}$ (Scherzer defocus)

$\Delta f=-200 \mathrm{~nm}$

$\Delta f=-400 \mathrm{~nm}$

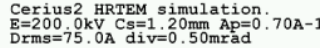
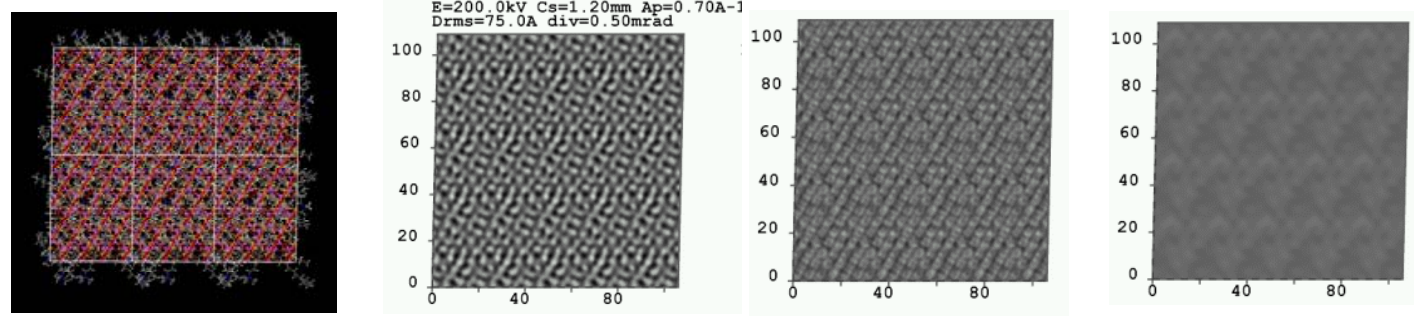

[001] orientation

Figure 2. Multislice simulations of image contrast at different values of objective lens defocus.
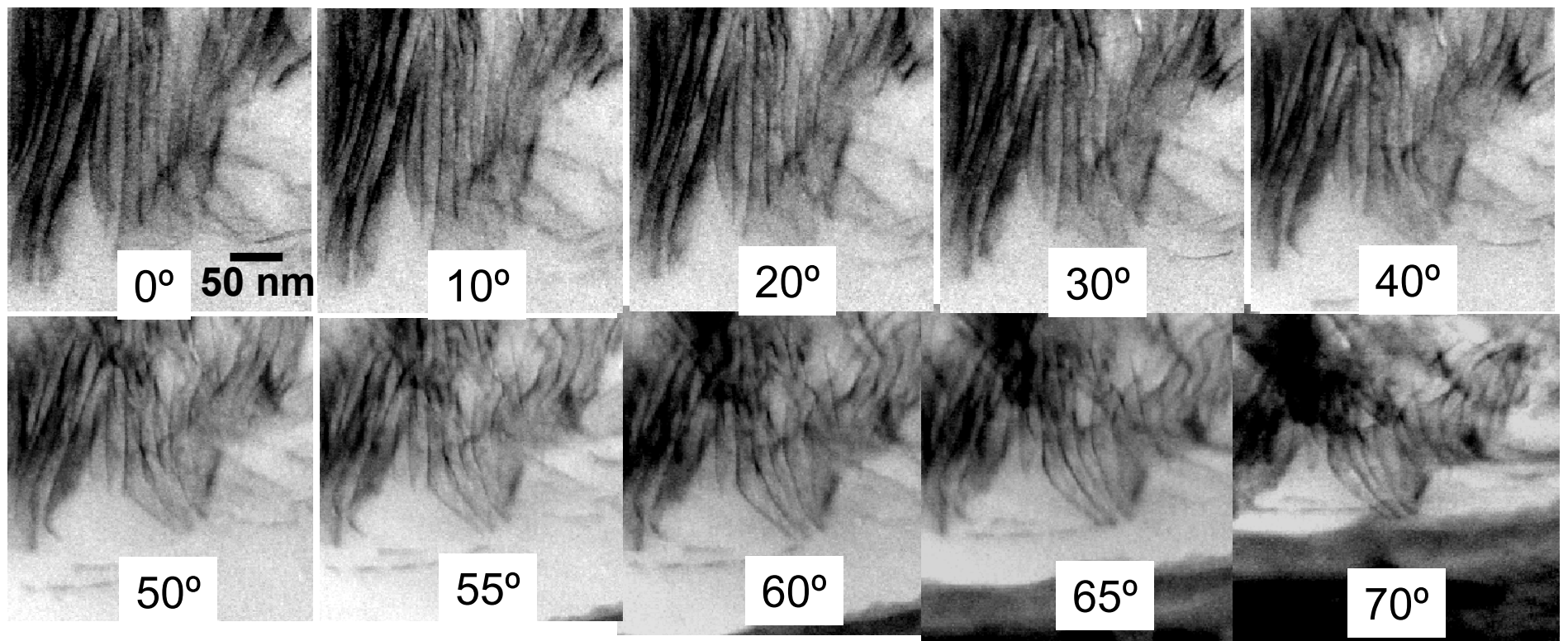

Figure 3. DF-STEM tilt series on an epoxy/MMT nanocomposite. 